\title{
Joint Weighted Least-Squares Estimation of Carrier-Frequency Offset and Timing Offset for OFDM Systems Over Multipath Fading Channels
}

\author{
Pei-Yun Tsai, Student Member, IEEE, Hsin-Yu Kang, and Tzi-Dar Chiueh, Senior Member, IEEE
}

\begin{abstract}
This paper presents an algorithm for joint estimation of carrier-frequency offset and timing offset for orthogonal frequency-division multiplexing (OFDM) systems in the tracking mode. The proposed weighted least-squares algorithm derives its estimates based on phase differences in the received pilot subcarrier signals between two symbols. Moreover, the optimal weights in two different channel conditions are derived. Both analysis and simulation show that the weighted least-squares algorithm can effectively and accurately estimate the carrier-frequency offset as well as the timing offset of OFDM signals in multipath fading channels.
\end{abstract}

Index Terms-Carrier-frequency offset, estimation, synchronization, timing offset, weighted least squares.

\section{INTRODUCTION}

$\mathbf{O}$ RTHOGONAL frequency-division multiplexing (OFDM) modulation offers an attractive solution to high-rate wired and wireless data access for its effectiveness against frequency-selective multipath fading using relatively simple frequency-domain equalization. In addition, OFDM also is very efficient in spectrum utilization, since the spectra of adjacent subcarriers overlap. Therefore, OFDM has been adopted in several communication standards, e.g., digital video broadcasting-terrestrial (DVB-T), very-high-speed digital subscriber line (VDSL), and IEEE 802.11a/g wireless local area network (LAN). However, it is also well known that OFDM systems are very sensitive to synchronization errors, which cause intercarrier interference (ICI) and degrade system performance.

All the OFDM standards mentioned before have dedicated pilot subcarriers to facilitate the synchronization tasks in the receivers. Numerous pilot-aided carrier-frequency and timingoffset estimation algorithms have been proposed [1]-[11]. Most utilized the phases of the received frequency-domain complex data at the pilot subcarriers. The phase shifts in the received complex data due to carrier-frequency offset are identical at all subcarriers in an OFDM symbol if the ICI is ignored. Classen [1], Kapoor [2], and Moose [3] have taken advantage of this fact in their estimation algorithms. The phases of the received

Manuscript received November 3, 2003; revised April 26, 2004, August 17, 2004 , and September 13, 2004. This work was supported in part by the National Science Council, Taiwan, under Grant NSC91-2219-E-002-022. This work was presented in part at the IEEE Vehicular Technology Conference (VTC), Cheju, Korea, Spring 2003. The review of this paper was coordinated by Prof. T. Lok.

The authors are with the Graduate Institute of Electronics Engineering and the Department of Electrical Engineering, National Taiwan University, Taipei 10617, Taiwan.

Digital Object Identifier 10.1109/TVT.2004.838891 pilot-subcarrier data were first extracted. In [1] and [2], the phase differences between all pilot-subcarrier signals and their respective counterparts in the previous symbol were computed. Then, the phase differences were averaged among all pilot subcarriers and/or over several OFDM symbols to estimate the carrier-frequency offset. On the other hand, a maximum likelihood (ML) method was adopted in [3] for the carrier-frequency offset estimation. In the ML estimator, the complex data at the pilot subcarriers, instead of their phases, were averaged. However, these algorithms may produce biased estimation when there exists timing offset, which occurs quite often in communication systems.

Timing offset (or sampling-clock offset), unlike carrier-frequency offset, causes phase shift that is proportional to the subcarrier index as well as the offset itself. A very popular class of schemes estimates the timing offset by computing a slope from the plot of measured pilot subcarrier phases versus pilot subcarrier indexes [4]-[7]. In [4], the slope was obtained by averaging over phase differences between pairs of adjacent pilot subcarriers. On the other hand, both the phase and magnitude of the pilot subcarriers in a single OFDM symbol were used in the slope calculation described in [5]. In [6] and [7], linear least-squares (LLS) estimation was adopted in the estimation of the slope. All four of these algorithms examined only the phases of the pilot subcarriers in one symbol, which were influenced by not only the timing offset, but also the frequency-selective fading. Therefore, the estimated timing offset can be far off. One possible solution is to take the phase differences between the pilot-subcarrier signals and their counterparts in the previous symbol so that the frequency-selective fading, being essentially the same in two symbols, is cancelled.

Joint estimation of carrier-frequency and timing offset has been studied extensively in [8]-[11]. In [8], the LLS method was adopted to obtain the best intercept and slope in the plot of pilot subcarrier signal phase versus pilot subcarrier index. However, only the additive white Gaussian noise (AWGN) channel was investigated in that study. Simoens [9] proposed an ML estimator and used the Taylor series expansion to get an analytical expression for the estimation. Both algorithms used phases of signals at pilot subcarriers in one OFDM symbol. Sliskovic [10] proposed to estimate the timing offset by computing the phase differences between pairs of adjacent pilot subcarriers, then computing the differences of those differences between two OFDM symbols and finally weighted averaging. The carrier-frequency offset was then estimated by first calculating the pilot subcarrier phase differences between two 
OFDM symbols, removing the contribution from the previously estimated timing offset, and finally weighted averaging. The concept of weighting was introduced and a weight was assigned to each pilot subcarrier during averaging, since the pilot subcarriers suffer from different levels of fading and, thus, may have a different signal-to-noise ratio (SNR). However, in this algorithm the accuracy of carrier-frequency offset estimation depends on not only the noise contained in the measurements, but also the accuracy of timing-offset estimation. In [11], one-dimensional (1-D) LLS estimation was applied to the plot of the pilot subcarrier phase difference between two adjacent symbols versus pilot subcarrier index. Another method used two-dimensional (2-D) LLS estimation over a frame consisting of several OFDM symbols.

In this paper, we present a weighted least-squares algorithm, also utilizing pilot subcarriers, to jointly estimate carrier-frequency and timing offsets in OFDM signals. In addition, we have derived the optimal weights for both the AWGN as well as the multipath fading channels. Both analysis and simulation results show that the proposed algorithm outperforms other algorithms.

The rest of this paper is organized as follows. The OFDM signals with both carrier and timing-synchronization errors are analyzed in Section II. The joint estimation based on a weighted least-squares algorithm is proposed and the optimal weights are derived in Section III. Also, the estimation performance of the proposed algorithm is analyzed and it is shown to approach the Cramér-Rao bound. In Section IV, the simulation results that demonstrate the advantages of the proposed algorithm are described. Finally, the conclusion is given in Section V.

\section{OFDM SIGNALS WITH SYNCHRONIZATION ERRORS}

An OFDM baseband symbol is generated by modulating $N$ complex data using the inverse discrete Fourier transform (IDFT) on $N$ subcarriers, as shown in Fig. 1. The subcarrier spacing is $1 / T$, where $T$ is the symbol duration. Note that in order to combat intersymbol interference (ISI), a cyclic prefix of $N_{g}$ samples is inserted at the beginning of each symbol. Therefore, each OFDM symbol has $N+N_{g}$ samples. Assume that the origin of the time axis aligns with the start of the cyclic prefix of the 0th symbol; then, the $n$th time-domain sample of the $i$ th transmitted symbol can be expressed as

$$
\begin{gathered}
d\left(i\left(N+N_{g}\right) T_{s}+N_{g} T_{s}+n T_{s}\right) \\
=d_{n, i}=\frac{1}{N} \sum_{k=-N / 2+1}^{N / 2} A_{k, i} e^{j 2 \pi n k / N}, \\
n=-N_{g}, \ldots, N-1
\end{gathered}
$$

where $d(t)$ is the transmitted signal, $T_{s}$ is the sample interval, and $A_{k, i}$ is the $k$ th complex data of the $i$ th symbol.

Assume that the received signal is corrupted in a multipath fading channel with a channel impulse response

$$
h(t)=\sum_{r} h_{r}(t) \cdot \delta\left(t-\tau_{r}(t)\right) .
$$

The gain and delay of the $r$ th path are denoted by $h_{r}(t)$ and $\tau_{r}(t)$, respectively. After convolving with the channel impulse

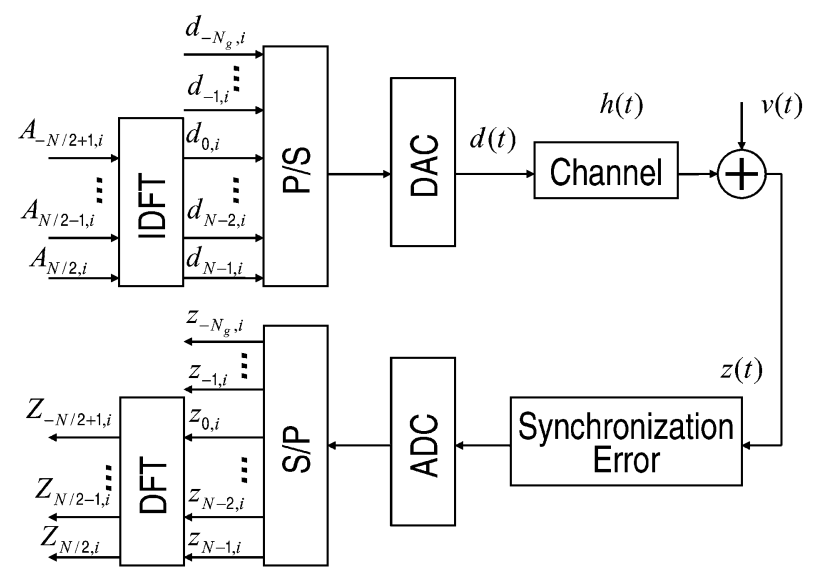

Fig. 1. System model of an OFDM communication system with $N$ subcarriers.

response and adding the channel noise $v(t)$, the received signal takes the form of

$$
z(t)=\sum_{r} h_{r}(t) \cdot d\left(t-\tau_{r}(t)\right)+v(t) .
$$

In addition to multipath channel fading, oscillator mismatch and the Doppler effect inflict the received signal with carrierfrequency and timing offsets. If some carrier-frequency offset $\Delta f$ and timing offset $\delta T_{s}$ exist, the $n$th received sample of the $i$ th symbol is given by

$$
\begin{array}{r}
z_{n, i}=\left.z(t) \cdot e^{j 2 \pi \Delta f t}\right|_{t=i\left(N+N_{g}\right)(1+\delta) T_{s}+N_{g}(1+\delta) T_{s}+n(1+\delta) T_{s}} \\
n=-N_{g}, \ldots, N-1 .
\end{array}
$$

Note that the sampling instant of the $n$th received sample of the $i$ th symbol is similar to that in (1) except that, due to the existence of the timing offset, $T_{s}$ is replaced by $(1+\delta) T_{s}$.

The receiver then drops the cyclic prefix and passes the $N$ samples of the symbol to an $N$-point discrete Fourier transform (DFT) block. Applying the DFT on $z_{n, i}$ and using (1) and (3), we have the received complex frequency-domain data on the $k$ th subcarrier of the $i$ th symbol $Z_{k, i}$ as

$$
\begin{aligned}
Z_{k, i}= & \sum_{n=0}^{N-1} z_{n, i} e^{-j 2 \pi n k / N} \\
= & e^{j 2 \pi\left(i N+i N_{g}+N_{g}\right)(1+\delta) \epsilon / N} \\
& \cdot \sum_{q=}^{N / 2} A_{q, i} H_{q} \cdot e^{j 2 \pi\left(i N+i N_{g}+N_{g}\right) \delta q / N} \\
& \cdot \frac{1}{N} \sum_{n=0}^{N-1} e^{-j 2 \pi n k / N} \cdot e^{j 2 \pi(1+\delta) n \epsilon / N} \\
& \cdot e^{j 2 \pi(1+\delta) q n / N}+V_{k, i} \\
= & \cdot \sum_{q=}^{j 2 \pi\left(i N+i N_{g}+N_{g}\right)(1+\delta) \epsilon / N} \\
& \cdot \frac{1}{N} \sum_{n=0}^{N-1} A_{q, i} H_{q} e^{j 2 \pi\left(i N+i N_{g}+N_{g}\right) \delta q / N}
\end{aligned}
$$

where $\epsilon=\Delta f \cdot T$ is the normalized carrier-frequency offset, $A_{k, i}$ and $V_{k, i}$ are the $k$ th transmitted subcarrier data, and the 
$k$ th received subcarrier noise component of the $i$ th symbol, respectively. $H_{k}$ represents the channel complex response at the $k$ th subcarrier. Also note that

$$
\phi_{q k}=(1+\delta) \cdot(\epsilon+q)-k .
$$

For clarity, $Z_{k, i}$ is further decomposed into three components: a signal term $S_{k, i}$; an interference term $I_{k, i}$; and a noise term $V_{k, i}$

$$
Z_{k, i}=S_{k, i}+I_{k, i}+V_{k, i} .
$$

Define the magnitude attenuation function

$$
s\left(\pi \phi_{q k}\right)=\frac{\sin \left(\pi \phi_{q k}\right)}{N \sin \left(\frac{\pi \phi_{q k}}{N}\right)} .
$$

Then, the signal term can be expressed as

$$
\begin{array}{r}
S_{k, i}=A_{k, i} H_{k} \cdot s\left(\pi \phi_{k k}\right) \cdot e^{j 2 \pi\left(i N+i N_{g}+N_{g}\right) \phi_{k k} / N} \\
\cdot e^{j \pi \phi_{k k}(1-(1 / N))}
\end{array}
$$

and the intercarrier interference term is given by

$$
\begin{aligned}
I_{k, i}= & \sum_{q=-N / 2+1, q \neq k}^{N / 2} A_{q, i} H_{q} \cdot s\left(\pi \phi_{q k}\right) \\
& \cdot e^{j 2 \pi\left(i N+i N_{g}+N_{g}\right) \phi_{q q} / N} \cdot e^{j \pi \phi_{q k}(1-(1 / N)) .}
\end{aligned}
$$

In the cases with small $\delta$ and $\epsilon, s\left(\pi \phi_{k k}\right)$ is close to 1 and $s\left(\pi \phi_{q k}\right)$ is close to zero for $q \neq k$. Therefore, the ICI term may be ignored in those cases.

The main impact of the carrier-frequency and timing offsets on the signal term can be summarized as the following. When both the carrier-frequency and timing offsets are present, $\phi_{k k}=$ $(1+\delta) \epsilon+\delta k$. Consequently, $S_{k, i} / H_{k}$ will be shifted in phase by

$$
\pi(\epsilon+\delta k+\delta \epsilon)\left(\frac{2\left(i N+i N_{g}+N_{g}\right)+N-1}{N}\right) .
$$

Consider the special case when only the carrier-frequency offset exists $(\delta=0)$. The phase shift is independent of $k$ and is identical in every subcarrier. On the other hand, if there is only the timing offset $(\epsilon=0)$, the phase shift is proportional to the subcarrier index $k$ as well as the timing offset itself.

\section{JOINT ESTIMATION OF CARRIER-FREQUENCY AND TIMING OFFSETS}

\section{A. Weighted Least-Squares Estimation}

In the AWGN channel, $H_{k}=1$ for all $k$ and $S_{k, i}$ is distorted in phase and amplitude caused by synchronization errors and noise. This effect is evident in Fig. 2, which illustrates the phases of subcarrier data in two adjacent OFDM symbols when they are distorted by noise, carrier-frequency offset, and timing offset. The carrier-frequency offset is 0.05 subcarrier spacing and the timing offset ratio $\delta$ is set to 100 parts per million (ppm). The received data contain ICI and noise; therefore, the extracted phases deviate from the two ideal straight lines. With the ex-

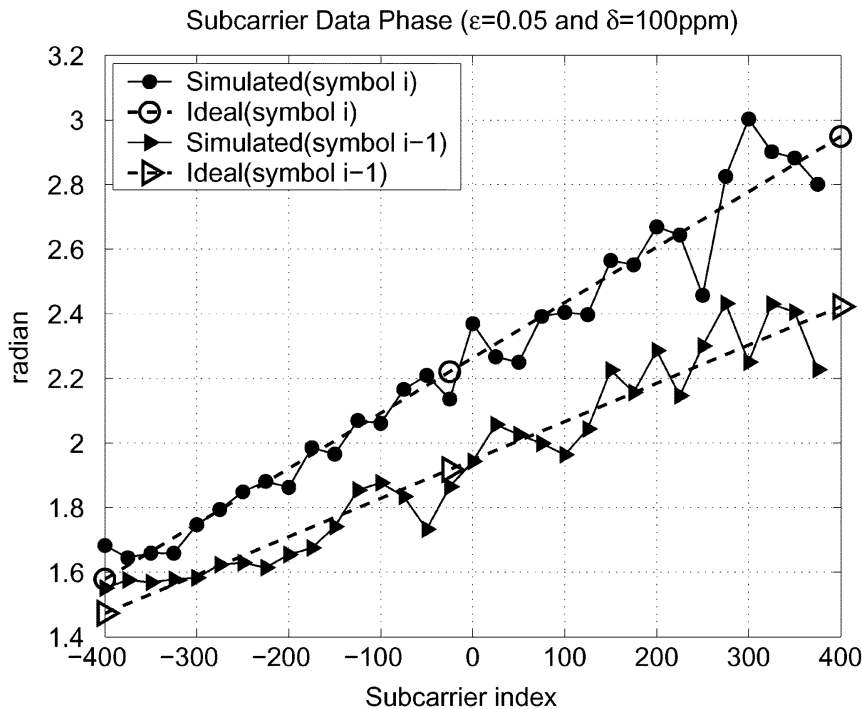

Fig. 2. Phases of subcarrier data in two OFDM symbols with $\epsilon=0.05, \delta=$ $100 \mathrm{ppm}$, and Gaussian noise.

tracted subcarrier data phases, the LLS method can estimate the two straight lines, from which the carrier-frequency and timing offsets can be derived.

In the multipath fading channel, the complex channel response $H_{k}$ distorts the received data in both phase and magnitude. Furthermore, signals on the deeply faded subcarriers have low SNR, while those on the subcarriers with little fading have high SNR. Obviously, the data phases of the subcarriers with higher SNR are more reliable than those with lower SNR in the estimation process. In order to increase the estimation accuracy, we need to consider the following:

- estimation based on the phase difference across two OFDM symbols can remove the common channel-phase response;

- weighting the data in each subcarrier is advantageous because data of deeply faded subcarriers should be assigned smaller weights to minimize their adverse effect on estimation accuracy;

- linear regression provides better performance since it can simultaneously find the best slope and intercept in terms of least-squared error.

In light of all the above considerations, we propose to jointly estimate the carrier-frequency and timing offsets by utilizing a weighted least-squares (WLS) algorithm.

Assume that $J$ pilots are inserted among $N$ subcarriers and that these pilot subcarrier indexes are denoted by $x_{j}, j=0,1, \ldots, J-1$. Pilot data are differentially encoded with a pseudonoise-sequence (PN-sequence) $C_{x_{j}, i} \in\{+1,-1\}$, which is known at the receiver, and $A_{x_{j}, i}=A_{x_{j}, i-1} C_{x_{j}, i}$. In order to apply the WLS estimation algorithm, we first compute the averaged phase difference between two consecutive OFDM symbols at the receiver

$$
y_{j}=\arg \left(\sum_{i} C_{x_{j}, i} Z_{x_{j}, i} Z_{x_{j}, i-1}^{*}\right)
$$


where $\arg (\cdot)$ is the phase of its argument and the summation is done over several symbols for averaging. Note that the multiplication of the PN-code $C_{x_{j}, i}$ is to sum the phase shifts between several pairs of symbol coherently.

From (11) and since $\delta \cdot \epsilon$ can be ignored, we have

$$
\begin{aligned}
y_{j} & =\arg \left(\sum_{i} C_{x_{j}, i} Z_{x_{j}, i} Z_{x_{j}, i-1}^{*}\right) \\
& =\arg \left(\sum_{i} C_{x_{j}, i} S_{x_{j}, i} S_{x_{j}, i-1}^{*}\right)+e_{j} \\
& =2 \pi \frac{N+N_{g}}{N}\left(\delta x_{j}+\epsilon\right)+e_{j} \\
& =2 \pi \frac{N+N_{g}}{N}\left[\begin{array}{ll}
x_{j} & 1
\end{array}\right]\left[\begin{array}{ll}
\delta & \epsilon
\end{array}\right]^{T}+e_{j}
\end{aligned}
$$

where $e_{j}$ is given by

$$
e_{j}=y_{j}-\arg \left(\sum_{i} C_{x_{j}, i} S_{x_{j}, i} S_{x_{j}, i-1}^{*}\right) .
$$

By stacking (14) for $j=0,1, \ldots, J-1$ and expressing them in vector form, we have

$$
\mathbf{y}=\mathbf{X b}+\mathbf{e}
$$

where

and

$$
\begin{aligned}
\mathbf{y} & =\left[\begin{array}{llll}
y_{0} & y_{1} & \cdots & y_{J-1}
\end{array}\right]^{T} \\
\mathbf{X} & =\left[\begin{array}{cccc}
x_{0} & x_{1} & \cdots & x_{J-1} \\
1 & 1 & 1 & 1
\end{array}\right]^{T} \\
\mathbf{b} & =2 \pi \frac{N+N_{g}}{N}\left[\begin{array}{ll}
\delta & \epsilon
\end{array}\right]^{T}
\end{aligned}
$$

$$
\mathbf{e}=\left[\begin{array}{ll}
e_{0} & e_{1} \ldots e_{J-1}
\end{array}\right]^{T}
$$

Assume that the channel is stationary during these symbols; the WLS estimate of $\mathbf{b}$ is given by [12]

$$
\hat{\mathbf{b}}=\left(\mathbf{X}^{T} \mathbf{W X}\right)^{-1} \mathbf{X}^{T} \mathbf{W} \mathbf{y}
$$

where $\mathbf{W}$ is the weight matrix in the WLS estimation algorithm. Then, the estimated carrier-frequency offset $\hat{\epsilon}$ and timing offset $\hat{\delta}$ can be obtained by

$$
\left[\begin{array}{ll}
\hat{\delta} & \hat{\epsilon}
\end{array}\right]^{T}=\frac{N \hat{\mathbf{b}}}{2 \pi\left(N+N_{g}\right)} .
$$

If we use

$$
\mathbf{W}=E\left\{\mathbf{e e}^{T}\right\}^{-1}
$$

then the WLS estimation has minimum variance in estimation error [12]. If we further assume that the error vector $\mathbf{e}$ is zero mean, then the WLS estimation is unbiased since

$$
E\{\hat{\mathbf{b}}\}=E\left\{\left(\mathbf{X}^{T} \mathbf{W} \mathbf{X}\right)^{-1} \mathbf{X}^{T} \mathbf{W}(\mathbf{X} \mathbf{b}+\mathbf{e})\right\}=\mathbf{b} .
$$

In this case, the WLS estimation provides the minimum mean squared estimation error. Moreover, the covariance matrix of the estimation error becomes

$$
\begin{aligned}
& E\left\{(\hat{\mathbf{b}}-\mathbf{b})(\hat{\mathbf{b}}-\mathbf{b})^{T}\right\} \\
& \quad=\left(\mathbf{X}^{T} \mathbf{W} \mathbf{X}\right)^{-1} \mathbf{X}^{T} \mathbf{W} E\left\{\mathbf{e} \mathbf{e}^{T}\right\} \mathbf{W}^{T} \mathbf{X}\left(\mathbf{X}^{T} \mathbf{W}^{T} \mathbf{X}\right)^{-1} \\
& \quad=\left(\mathbf{X}^{T} \mathbf{W} \mathbf{X}\right)^{-1}
\end{aligned}
$$

\section{B. Derivation of the Optimal Weights}

According to (18), we have to evaluate the error-phase covariance matrix $E\left\{\mathbf{e e}^{T}\right\}$ in order to obtain the optimal weights. However, since it is difficult to derive the error-phase covariance matrix directly, we will calculate the statistics of the received signal phasor instead and then use numerical approximation to obtain the error-phase covariance matrix $E\left\{\mathbf{e e}^{T}\right\}$. From (12) and (14), we first work on the statistics of $C_{x_{j}, i} Z_{x_{j}, i} Z_{x_{j}, i-1}^{*}$, then derive the statistics of $y_{j}$, those of $e_{j}$, and finally the crosscorrelation of $e_{j}$ and $e_{l}$.

The phasor $C_{x_{j}, i} Z_{x_{j}, i} Z_{x_{j}, i-1}^{*}$ can be expressed as

$$
\begin{aligned}
& C_{x_{j}, i} Z_{x_{j}, i} Z_{x_{j}, i-1}^{*}=C_{x_{j}, i} S_{x_{j}, i} S_{x_{j}, i-1}^{*}+C_{x_{j}, i} S_{x_{j}, i} I_{x_{j}, i-1}^{*} \\
& \quad+C_{x_{j}, i} S_{x_{j}, i} V_{x_{j}, i-1}^{*}+C_{x_{j}, i} I_{x_{j}, i} S_{x_{j}, i-1}^{*} \\
& \quad+C_{x_{j}, i} I_{x_{j}, i} I_{x_{j}, i-1}^{*}+C_{x_{j}, i} I_{x_{j}, i} V_{x_{j}, i-1}^{*} \\
& \quad+C_{x_{j}, i} V_{x_{j}, i} S_{x_{j}, i-1}^{*}+C_{x_{j}, i} V_{x_{j}, i} I_{x_{j}, i-1}^{*} \\
& \quad+C_{x_{j}, i} V_{x_{j}, i} V_{x_{j}, i-1}^{*}
\end{aligned}
$$

The noises $V_{x_{j}, i}$ and $V_{x_{j}, i-1}$ are zero mean and independent of the signal and ICI; thus, all terms in (20) that involve $V_{x_{j}, i}$ and $V_{x_{j}, i-1}$ have zero expectation. Furthermore, assume that the data $A_{k, i}$ are independent and identically distributed (i.i.d.) random variables with the following statistics:

$$
\begin{aligned}
E\left\{A_{k, i}\right\} & =0 \\
E\left\{A_{k, i} A_{p, i}^{*}\right\} & =E_{s} \delta_{k p}
\end{aligned}
$$

where $E_{s}$ is the average subcarrier energy. Since the pilot data are differentially coded with a PN-sequence, thus

$$
E\left\{C_{x_{j}, i} A_{x_{j}, i} A_{k, i-1}^{*}\right\}=E_{s} \delta_{x_{j} k}
$$

It can then be shown that the expectation of the remaining terms are given by (see Appendix A for details)

$$
\begin{aligned}
E & \left\{C_{x_{j}, i} S_{x_{j}, i} S_{x_{j}, i-1}^{*}\right\} \\
& =E_{s}\left|H_{x_{j}}\right|^{2} s^{2}\left(\pi \phi_{x_{j} x_{j}}\right) e^{j 2 \pi\left(N+N_{g}\right) \phi_{x_{j} x_{j}} / N}
\end{aligned}
$$

and

$$
\begin{gathered}
E\left\{C_{x_{j}, i} I_{x_{j}, i} I_{x_{j}, i-1}^{*}\right\}=E\left\{C_{x_{j}, i} S_{x_{j}, i} I_{x_{j}, i-1}^{*}\right\} \\
=E\left\{C_{x_{j}, i} I_{x_{j}, i} S_{x_{j}, i-1}^{*}\right\}=0 .
\end{gathered}
$$


TABLE I

DERIVED WLS ESTIMATION WEIGHTS IN TWO CASES

\begin{tabular}{c|c}
\hline Channel condition & $w_{j}$ \\
\hline \hline Multipath channel with noise & $\frac{I E_{s}\left|H_{x_{j}}\right|^{2}}{\sigma_{v}^{2}}$ \\
\hline Multipath channel with noise and ICI & $\frac{I E_{s}\left|H_{x_{j}}\right|^{2} s^{2}\left(\pi \phi_{x_{j} x_{j}}\right)}{\sigma_{v}^{2}+\sum_{k \neq x_{j}} E_{s}\left|H_{k}\right|^{2} s^{2}\left(\pi \phi_{k x_{j}}\right)}$ \\
\hline
\end{tabular}

Note that, due to the PN sequence, the correlation of the two ICI terms and that of the signal term and either ICI term vanish. Hence

$$
\begin{aligned}
E & \left\{C_{x_{j}, i} Z_{x_{j}, i} Z_{x_{j}, i-1}^{*}\right\} \\
& =E\left\{C_{x_{j}, i} S_{x_{j}, i} S_{x_{j}, i-1}^{*}\right\} \\
& =E_{s}\left|H_{x_{j}}\right|^{2} s^{2}\left(\pi \phi_{x_{j} x_{j}}\right) e^{j 2 \pi\left(N+N_{g}\right) \phi_{x_{j} x_{j}} / N} .
\end{aligned}
$$

Next, we want to compute the variance of $C_{x_{j}, i} Z_{x_{j}, i} Z_{x_{j}, i-1}^{*}$. From (21) and (22), we have

$$
E\left\{A_{k, i} A_{p, i}^{*} A_{q, i-1}^{*} A_{r, i-1}\right\}=E_{s}^{2} \delta_{k p} \delta_{q r}
$$

and

$$
E\left\{C_{x_{j}, i} A_{x_{j}, i} A_{k, i-1}^{*} C_{x_{l}, i} A_{x_{l}, i}^{*} A_{q, i-1}\right\}=E_{s}^{2} \delta_{x_{j} k} \delta_{x_{l} q} .
$$

In Appendix B, we show that the covariance of any two terms in (20) is zero. Thus, the variance of $C_{x_{j}, i} Z_{x_{j}, i} Z_{x_{j}, i-1}^{*}$ is the sum of the variances of individual terms in (20). Since $C_{x_{j}, i} S_{x_{j}, i} S_{x_{j}, i-1}^{*}$ is a constant, thus its variance is zero. The variances of the remaining eight terms are derived in Appendix B. In most cases, when the noise and carrier-frequency and timing offsets are within their respective normal ranges, the variance of $C_{x_{j}, i} Z_{x_{j}, i} Z_{x_{j}, i-1}^{*}$ is dominated by the variances of those terms with either $S_{x_{j}, i}$ or $S_{x_{j}, i-1}$. For instance, if the SNR is greater than $18 \mathrm{~dB},-0.1<\epsilon<0.1$, and $\delta=20 \mathrm{ppm}$, then the sum of the variances of those terms that do not involve either $S_{x_{j}, i}$ or $S_{x_{j}, i-1}$ is less than $3 \%$ of the total amount. Therefore, we are left with four variance terms, as follows:

$$
\begin{aligned}
& \operatorname{Var}\left\{C_{x_{j}, i} S_{x_{j}, i} I_{x_{j}, i-1}^{*}\right\}=\operatorname{Var}\left\{C_{x_{j}, i} I_{x_{j}, i} S_{x_{j}, i-1}^{*}\right\} \\
& =E_{s}^{2}\left|H_{x_{j}}\right|^{2} s^{2}\left(\pi \phi_{x_{j} x_{j}}\right) \sum_{k \neq x_{j}}\left|H_{k}\right|^{2} s^{2}\left(\pi \phi_{k x_{j}}\right) \\
& \operatorname{Var}\left\{C_{x_{j}, i} S_{x_{j}, i} V_{x_{j}, i-1}^{*}\right\}=\operatorname{Var}\left\{C_{x_{j}, i} V_{x_{j}, i} S_{x_{j}, i-1}^{*}\right\} \\
& \quad=E_{s}\left|H_{x_{j}}\right|^{2} s^{2}\left(\pi \phi_{x_{j} x_{j}}\right) \sigma_{v}^{2}
\end{aligned}
$$

where $\sigma_{v}^{2}$ is the variance of $V_{x_{j}, i}$.

Note that since $A_{k, i}$ and $V_{k, i}$ are stationary, so is $C_{x_{j}, i} Z_{x_{j}, i} Z_{x_{j}, i-1}^{*}$. Therefore, the random variables $C_{x_{j}, i} Z_{x_{j}, i} Z_{x_{j}, i-1}^{*}$ for several different $i$ are i.i.d. Applying the Central Limit Theorem, one has that $\sum_{i} C_{x_{j}, i} Z_{x_{j}, i} Z_{x_{j}, i-1}^{*}$ can be approximated by a complex Gaussian random variable with mean

$$
\mu=I E_{s}\left|H_{x_{j}}\right|^{2} s^{2}\left(\pi \phi_{x_{j} x_{j}}\right) e^{j 2 \pi\left(N+N_{g}\right) \phi_{x_{j} x_{j}} / N}
$$

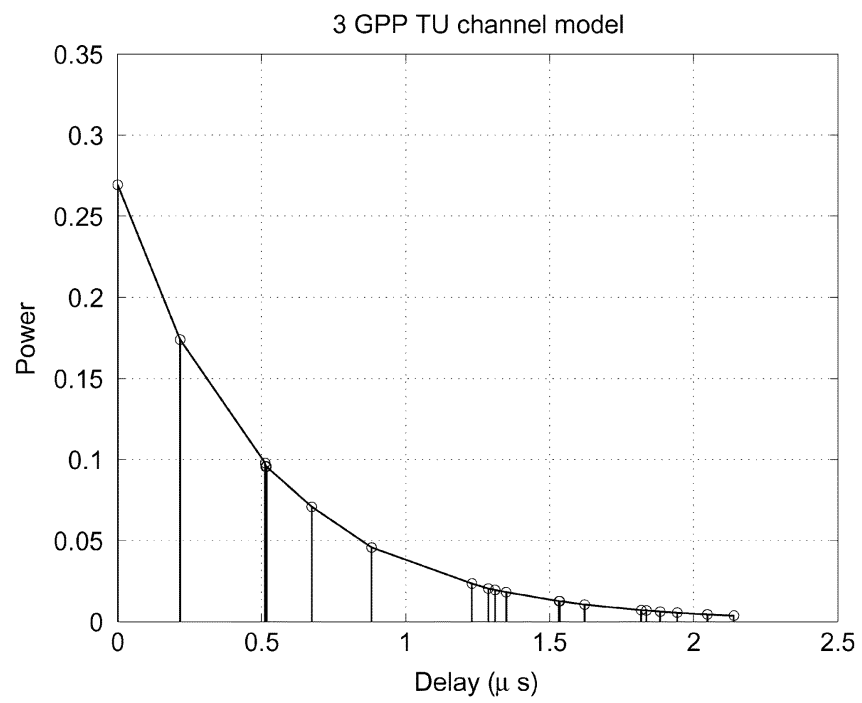

Fig. 3. 3GPP channel power delay profile in a typical urban scenario.

and variance

$$
\begin{aligned}
\sigma^{2} \approx 2 I E_{s}\left|H_{x_{j}}\right|^{2} & s^{2}\left(\pi \phi_{x_{j} x_{j}}\right) \\
& \times\left(\sum_{k \neq x_{j}} E_{s}\left|H_{k}\right|^{2} s^{2}\left(\pi \phi_{k x_{j}}\right)+\sigma_{v}^{2}\right)
\end{aligned}
$$

where $I$ is the number of symbols in the summand.

Define $r_{j}=\left|\sum_{i} C_{x_{j}, i} Z_{x_{j}, i} Z_{x_{j}, i-1}^{*}\right|$; then, the joint probability density function (pdf) of $r_{j}$ and $y_{j}$ is given by

$$
\begin{aligned}
p_{r_{j}, y_{j}}\left(r_{j}, y_{j}\right) & =\frac{r_{j}}{\pi \sigma^{2}} \\
\cdot \exp & {\left[-\left(\frac{r_{j}^{2}-2 r_{j} \mu_{r_{j}} \cos \left(y_{j}-\mu_{y_{j}}\right)+\mu_{r_{j}}^{2}}{\sigma^{2}}\right)\right] }
\end{aligned}
$$

where $\mu_{r_{j}}=I E_{s}\left|H_{x_{j}}\right|^{2} s^{2}\left(\pi \phi_{x_{j} x_{j}}\right)$ and $\mu_{y_{j}}=2 \pi(N+$ $\left.N_{g}\right) \phi_{x_{j} x_{j}} / N$. The pdf of $y_{j}$ can be obtained by integrating $r_{j}$ [13], [14]

$$
\begin{aligned}
p_{y_{j}}\left(y_{j}\right)= & \frac{1}{2 \pi} \exp \left(-\frac{\mu_{r_{j}}^{2}}{\sigma^{2}}\right) \\
& +\frac{1}{\sqrt{\pi}} \frac{\mu_{r_{j}} \cos \left(y_{j}-\mu_{y_{j}}\right)}{\sigma} \\
& \cdot \exp \left(-\frac{\mu_{r_{j}}^{2} \sin ^{2}\left(y_{j}-\mu_{y_{j}}\right)}{\sigma^{2}}\right) \\
& \cdot Q\left(-\frac{\sqrt{2} \mu_{r_{j}} \cos \left(y_{j}-\mu_{y_{j}}\right)}{\sigma}\right)
\end{aligned}
$$


where $Q(x)=((1) /(\sqrt{2 \pi})) \int_{x}^{\infty} e^{-t^{2} / 2} d t$. If the signal-to-interference-plus-noise ratio (SINR) is high, i.e., $\mu_{r_{j}}^{2} \gg \sigma^{2}$ and $y_{j}-\mu_{y_{j}} \approx 0$, then the first term in (28) can be neglected and it can be approximated by a Gaussian distribution with the density function [13], [14]

$$
p_{y_{j}}\left(y_{j}\right) \approx \frac{1}{\sqrt{\pi \sigma^{2} / \mu_{r_{j}}^{2}}} \exp \left(-\frac{\left(y_{j}-\mu_{y_{j}}\right)^{2} \mu_{r_{j}}^{2}}{\sigma^{2}}\right) .
$$

The mean and variance of $y_{j}$ are $\mu_{y_{j}}$ and $\sigma^{2} /\left(2 \mu_{r_{j}}^{2}\right)$, respectively. Denote the variance of $e_{j}$ as $\sigma_{e_{j}}^{2}$. Since $E\left\{e_{j}\right\}=0$ from (23), thus

$$
\sigma_{e_{j}}^{2}=E\left\{e_{j} e_{j}\right\}=\operatorname{Var}\left\{y_{j}\right\}=\sigma^{2} /\left(2 \mu_{r_{j}}^{2}\right) .
$$

With the diagonal terms in $E\left\{\mathbf{e e}^{T}\right\}$ at hand, we then turn to the off-diagonal terms $E\left\{e_{j} e_{l}\right\}$ for $j \neq l$. Again, it is easier to compute the covariance of the phasors $\sum_{i} C_{x_{j}, i} Z_{x_{j}, i} Z_{x_{j}, i-1}^{*}$ and $\sum_{i} C_{x_{l}, i} Z_{x_{l}, i} Z_{x_{l}, i-1}^{*}$ instead of directly computing $E\left\{e_{j} e_{l}\right\}$. The detailed derivations are given in Appendix C. The covariance of the two phasors is much smaller than either of the two variances. For instance, in the case of 30-dB SNR with $\epsilon=0.05$ and $\delta=5 \mathrm{ppm}$, the covariance is five order less than either variance. Accordingly, all the off-diagonal terms in $E\left\{\mathbf{e e}^{T}\right\}$ can be ignored.

With this simplification, the weight matrix in (18) is reduced to a diagonal matrix whose $j$ th diagonal component, denoted as $w_{j}$, is given by

$$
w_{j}=\sigma_{e_{j}}^{-2} .
$$

Using (30) in the high-SINR case, we can approximate $w_{j}$ by

$$
w_{j} \approx \frac{2 \mu_{r_{j}}^{2}}{\sigma^{2}} \approx \frac{I E_{s}\left|H_{x_{j}}\right|^{2} s^{2}\left(\pi \phi_{x_{j} x_{j}}\right)}{\sigma_{v}^{2}+\sum_{k \neq x_{j}} E_{s}\left|H_{k}\right|^{2} s^{2}\left(\pi \phi_{k x_{j}}\right)} .
$$

Moreover, in the case when the residual synchronization error is so small that we have $s\left(\pi \phi_{x_{j} x_{j}}\right) \approx 1$ and $s\left(\pi \phi_{k x_{j}}\right) \approx 0$ for $k \neq x_{j}$, the ICI term can be neglected when compared to the noise term. As a result, $w_{j}$ is simplified to

$$
w_{j} \approx \frac{2 \mu_{r_{j}}^{2}}{\sigma^{2}} \approx \frac{I E_{s}\left|H_{x_{j}}\right|^{2}}{\sigma_{v}^{2}} .
$$

The derived optimal weights for WLS estimation in both cases are summarized in Table I. The formulas indicate that the weights depend on the channel gains of the relevant subcarriers $\left(H_{x_{j}}\right.$ and $\left.H_{k}\right)$ and the magnitude attenuation factor $s\left(\pi \phi_{k x_{j}}\right)$, which in turn depends on the previous estimates of the synchronization errors.

With the approximation that the weight matrix $\mathbf{W}$ is a diagonal matrix, the estimation of carrier-frequency offset $\hat{\epsilon}$ and the timing offset $\hat{\delta}$ can be derived by simplifying (16) and we have (34) and (35), shown at the bottom of the page. In addition, the error covariance matrix is given by

$$
\left(\mathbf{X}^{T} \mathbf{W X}\right)^{-1}=\left[\begin{array}{cc}
\sum_{j=0}^{J-1} w_{j} x_{j}^{2} & \sum_{j=0}^{J-1} w_{j} x_{j} \\
\sum_{j=0}^{J-1} w_{j} x_{j} & \sum_{j=0}^{J-1} w_{j}
\end{array}\right]^{-1}
$$

Hence, the mean squared error (mse) of the estimated carrierfrequency offset $\hat{\epsilon}$ and the estimated timing offset $\hat{\delta}$, respectively, can be given by (37) and (38) at the bottom of the page. Furthermore, in most cases when $\mu_{r_{j}}^{2} \gg \sigma^{2}$ and $y_{j}-\mu_{y_{j}} \approx 0$, the observation $\mathbf{y}$ is approximately Gaussian distributed with mean $\mathbf{X b}$ and variance $E\left\{\mathbf{e e}^{T}\right\}$. Accordingly, the WLS estimator is the ML estimator that achieves the Cramér-Rao bound [12].

Finally, in a special case when all the weights in the WLS estimation are equal, the WLS estimation is equivalent to the LLS estimation [8]. When only carrier-frequency offset exits,

and

$$
\hat{\epsilon}=\frac{\left(\sum_{j=0}^{J-1} w_{j} x_{j}^{2}\right)\left(\sum_{j=0}^{J-1} w_{j} y_{j}\right)-\left(\sum_{j=0}^{J-1} w_{j} x_{j}\right)\left(\sum_{j=0}^{J-1} w_{j} y_{j} x_{j}\right)}{\left(2 \pi \frac{N+N_{g}}{N}\right)\left[\left(\sum_{j=0}^{J-1} w_{j}\right)\left(\sum_{j=0}^{J-1} w_{j} x_{j}^{2}\right)-\left(\sum_{j=0}^{J-1} w_{j} x_{j}\right)^{2}\right]}
$$

$$
\hat{\delta}=\frac{\left(\sum_{j=0}^{J-1} w_{j}\right)\left(\sum_{j=0}^{J-1} w_{j} y_{j} x_{j}\right)-\left(\sum_{j=0}^{J-1} w_{j} x_{j}\right)\left(\sum_{j=0}^{J-1} w_{j} y_{j}\right)}{\left(2 \pi \frac{N+N_{g}}{N}\right)\left[\left(\sum_{j=0}^{J-1} w_{j}\right)\left(\sum_{j=0}^{J-1} w_{j} x_{j}^{2}\right)-\left(\sum_{j=0}^{J-1} w_{j} x_{j}\right)^{2}\right]} .
$$

and

$$
\sigma_{\hat{\epsilon}}^{2}=\frac{\sum_{j=0}^{J-1} w_{j} x_{j}^{2}}{\left(2 \pi \frac{N+N_{g}}{N}\right)^{2} \cdot\left[\left(\sum_{j=0}^{J-1} w_{j}\right)\left(\sum_{j=0}^{J-1} w_{j} x_{j}^{2}\right)-\left(\sum_{j=0}^{J-1} w_{j} x_{j}\right)^{2}\right]}
$$




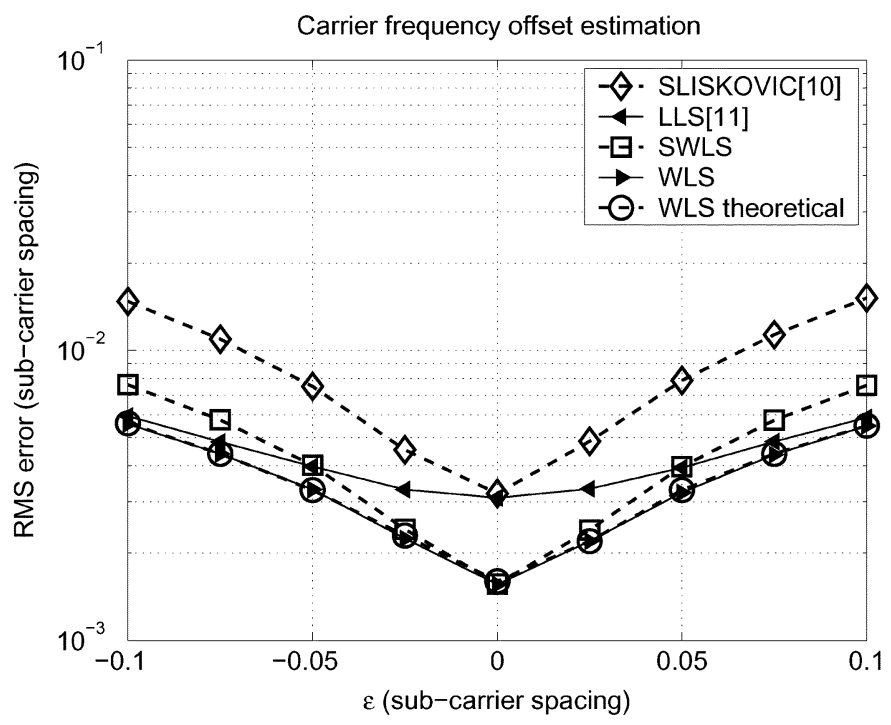

(a)

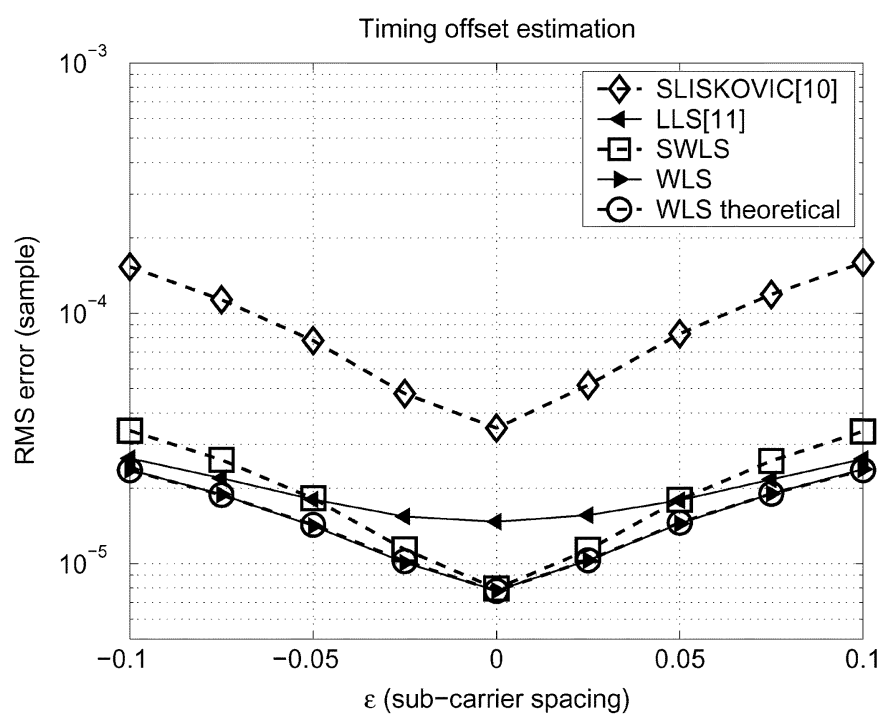

(b)

Fig. 4. (a) Carrier-frequency offset estimation rms errors and (b) timing offset estimation rms errors versus various $\epsilon$ by different algorithms in stationary multipath fading channel. The SNR is at $24 \mathrm{~dB}$ and the timing offset is -20 ppm.

the WLS estimation boils down to estimate only the intercept of a horizontal line and the estimated $\epsilon$ is given by

$$
\hat{\epsilon}=\frac{\sum_{j=0}^{J-1} w_{j} y_{j}}{\left(2 \pi \frac{N+N_{g}}{N}\right) \sum_{j=0}^{J-1} w_{j}} .
$$

Conversely, if there is no carrier-frequency offset, only the slope of a line passing through the origin needs be estimated and the WLS estimate on $\delta$ is given by

$$
\hat{\delta}=\frac{\sum_{j=0}^{J-1} w_{j} x_{j} y_{j}}{\left(2 \pi \frac{N+N_{g}}{N}\right) \sum_{j=0}^{J-1} w_{j} x_{j}^{2}}
$$

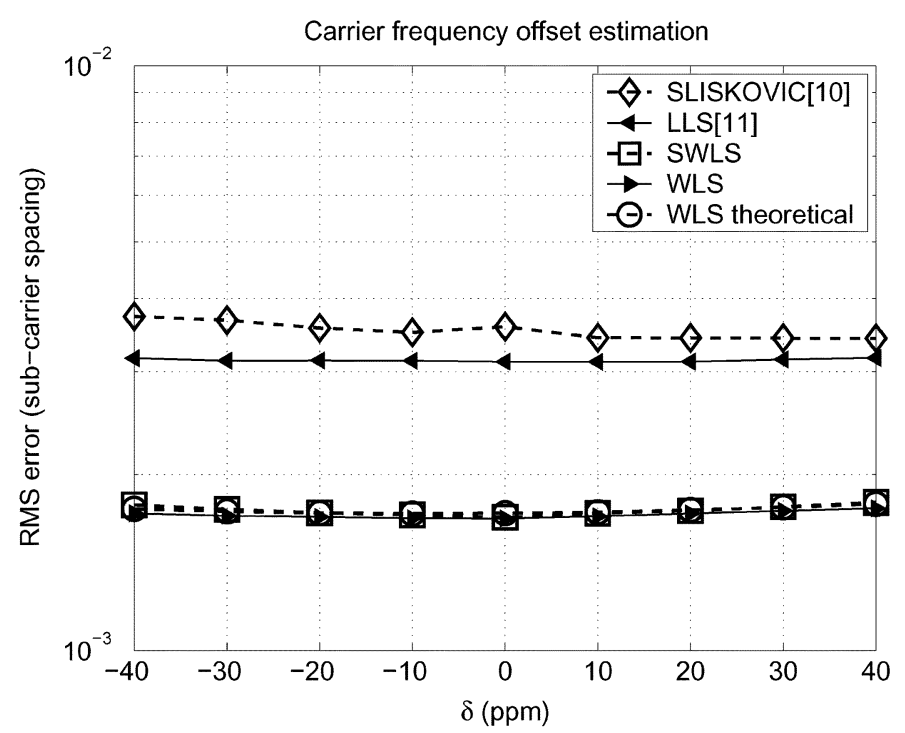

(a)

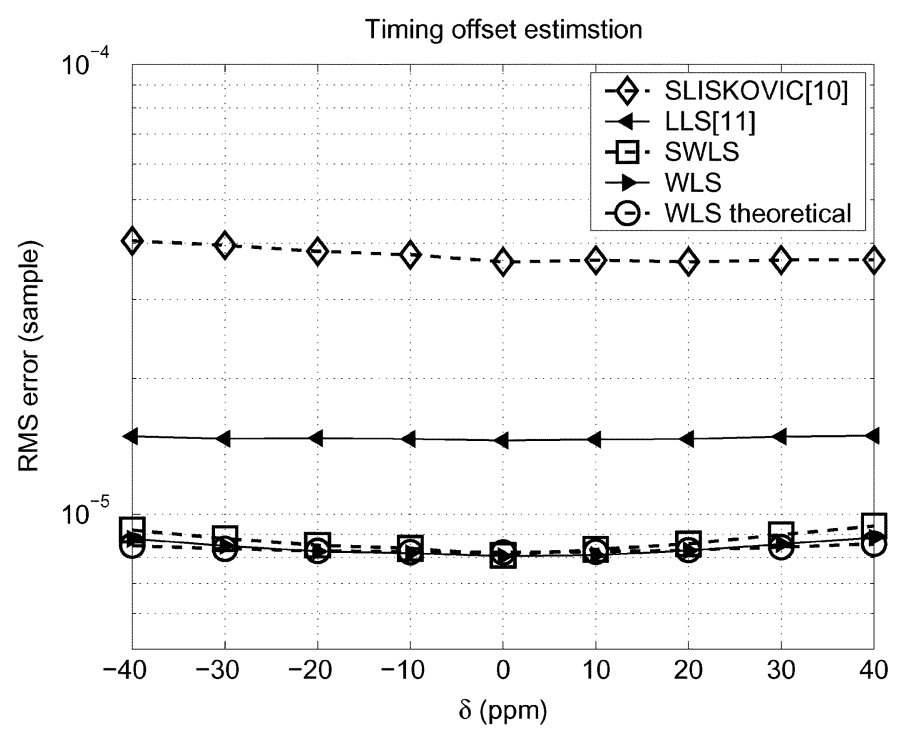

(b)

Fig. 5. (a) Carrier-frequency offset estimation rms errors and (b) timing offset estimation rms errors versus various $\delta$ by different algorithms in stationary multipath fading channel. The SNR is at $24 \mathrm{~dB}$ and the carrier-frequency offset is $\epsilon=0.01$.

\section{SimUlATION AND DisCUSSION}

In the simulation, we used an OFDM system that has a total of 1024 subcarriers, 224 of which are unused and work as guard bands on both ends of the signal band. There are 32 pilot subcarriers, evenly spaced across the entire 800 subcarriers. The guard interval is 64 samples long and the sampling frequency is 5.76 $\mathrm{MHz}$. Since we are interested in the case when both carrier-frequency and timing offsets are present, we simulate only joint estimation algorithms. We used the multipath channel model with the power delay profile in a typical urban scenario, where a large number of paths ensure that correlation properties in the frequency domain are realistic [16]. This profile is shown in Fig. 3.

In Fig. 4, we illustrate the simulated root-mean-squared (rms) estimation errors of several joint estimation algorithms in a stationary channel for different levels of carrier-frequency offset. 


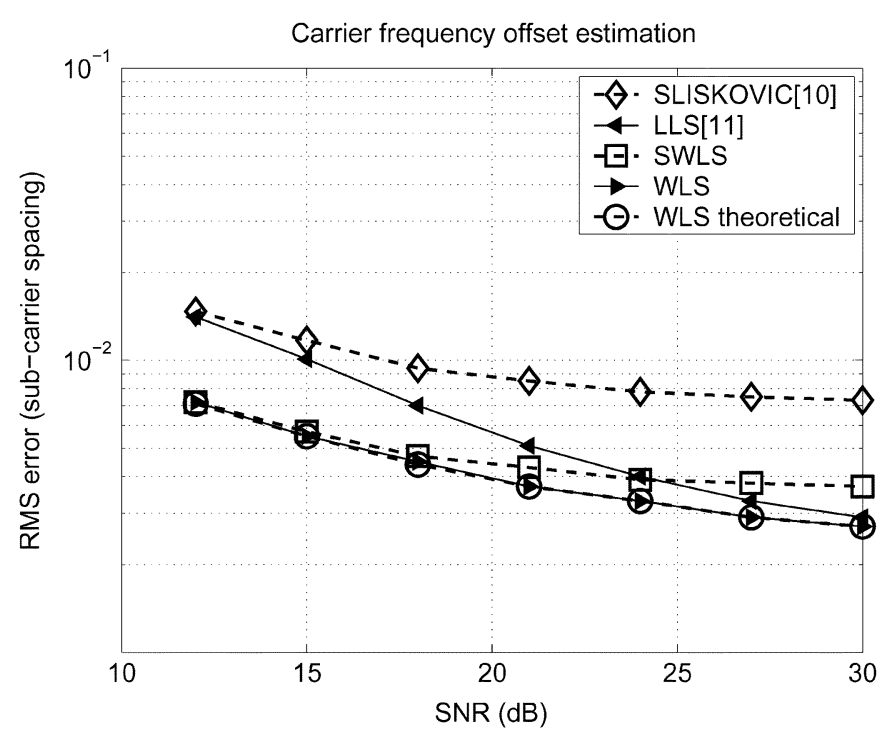

(a)

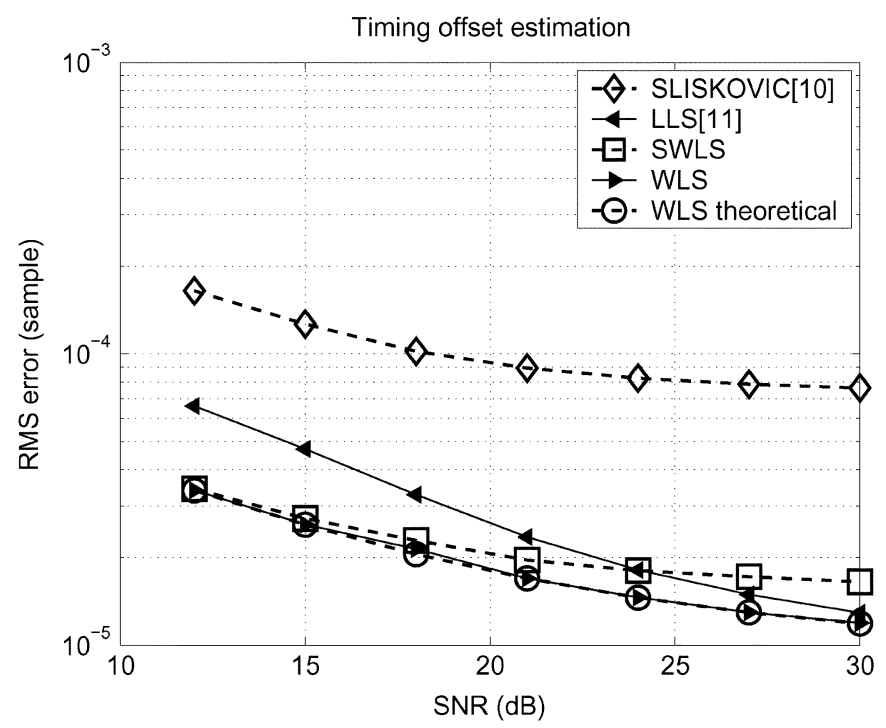

(b)

Fig. 6. (a) Carrier-frequency offset estimation rms errors and (b) timing offset estimation rms errors versus SNR by different algorithms in stationary multipath fading channel. The carrier-frequency offset and the timing offset are $\epsilon=0.05$ and $\delta=-20 \mathrm{ppm}$.

Similarly, the performances of the joint estimation algorithms under different levels of timing offset are depicted in Fig. 5. In these two figures, the curves labeled with "WLS" use the optimal weights listed in (32) and the rms errors are simulated results, while the curves labeled with "WLS theoretical" are computed using (37) and (38). Another simplified case labeled as "SWLS" uses the simplified weights, which are $\left|\hat{H}_{x_{j}}\right|^{2}, j=$ $0, \ldots, J-1$. In Fig. 4 , in addition to carrier-frequency offset, a constant timing offset of $\delta=-20 \mathrm{ppm}$ is injected and the SNR is fixed at $24 \mathrm{~dB}$. We see that the rms errors of the estimation become large when the magnitude of carrier-frequency offset increases. However, the WLS estimator has by far the best performance. Even the simplified estimator, SWLS, has better performance than [10] and [11] in most cases. As the carrier-frequency offset increases beyond an absolute value of 0.05 , the SWLS

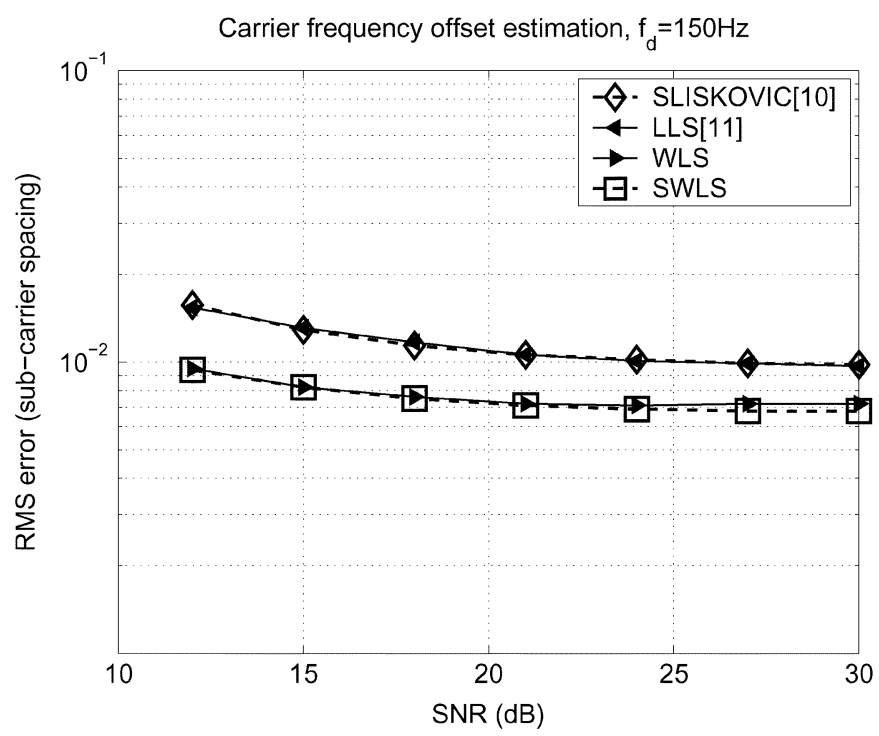

(a)

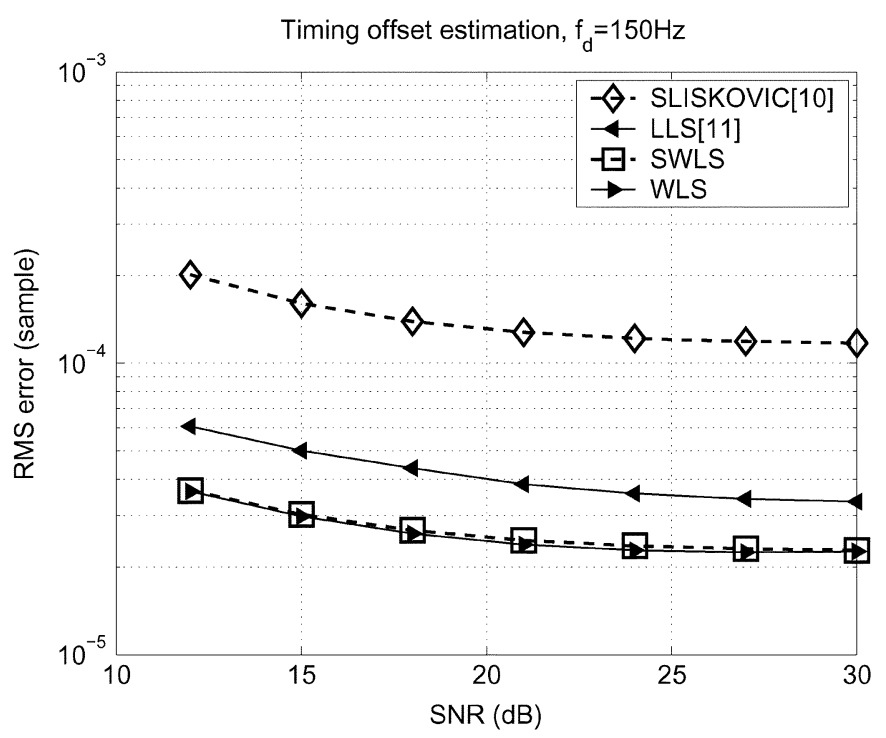

(b)

Fig. 7. (a) Carrier-frequency offset estimation rms errors and (b) timing offset estimation rms errors versus SNR by different algorithms in fast-fading multipath channels.

estimator suffers from insurmountable level of ICI, making it poorer than the LLS estimator.

Similarly, the performances of the joint estimation algorithms under different levels of timing offset are depicted in Fig. 5. In these simulations, the SNR is set to $24 \mathrm{~dB}$ and a carrier-frequency offset of $\epsilon=0.01$ is used. Relatively speaking, the timing offset usually incurs a much smaller level of ICI than the carrier-frequency offset. So, the rms estimation error curves are almost flat in the range of interest. As the ICI is negligible, the three cruves "SWLS," "WLS," and "WLS theoretical" have almost identical performance. Again, it is clear that the WLS algorithms outperform other joint estimation algorithms.

Next, a carrier-frequency error $\epsilon=0.05$ and a timing error $\delta=-20 \mathrm{ppm}$ are introduced. The rms errors of the joint carrier-frequency offset estimation and timing offset estimation algorithms under stationary channel condition versus various 


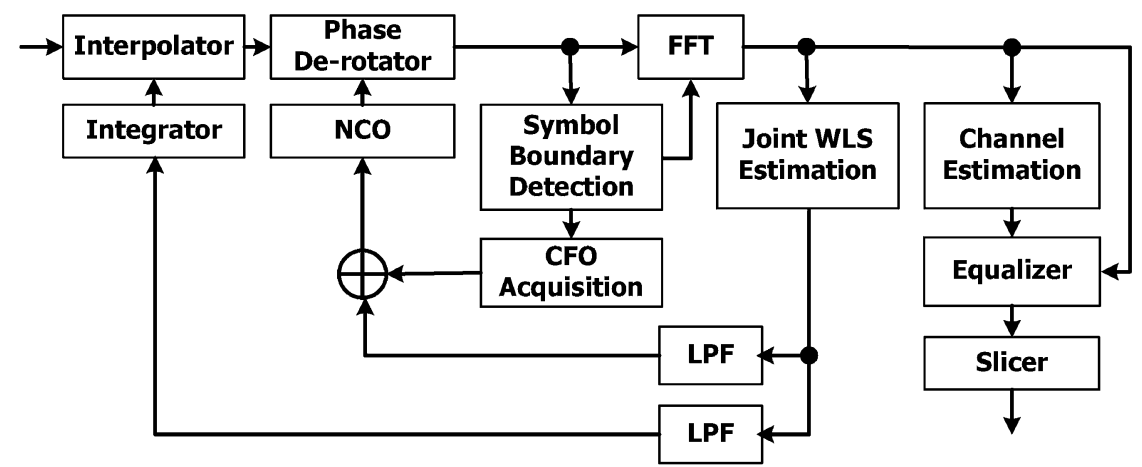

Fig. 8. Receiver architecture used in system simulation.

SNR levels are depicted in Fig. 6. We see that the Sliskovic's algorithm is poor in estimating both offsets and that the LLS estimation performs poorly in low-SNR cases because of equal weighting, yet its performance improves as the SNR increases. When the SNR is low, the SWLS algorithm, which considers noises only, has comparable performance as the WLS algorithm. This is because, in low-SNR cases, the noises overwhelm ICI and the two algorithms use practically the same weights. On the other hand, when the SNR is high, ICI can be a major impairment. Thus, the WLS algorithm, using the optimal weights that consider the ICI terms, outperforms the SWLS algorithm.

In another simulation with a fast fading channel, we used a Doppler frequency $\left(f_{d}\right)$ of $150 \mathrm{~Hz}$. The simulated estimation rms errors by four joint estimators are depicted in Fig. 7 . The performance of these estimators all deteriorate and the Doppler effect hurts these estimators in two main aspects: one is the extra ICI induced by the channel variation within one symbol and the other is the fluctuation in the complex channel gains. Because of these two extra impairments, the estimation errors in the dynamic channel have floors at levels equivalent to those of the stationary channel case with an SNR of about 15-17 dB. In [17], more comparisons of the proposed WLS method with other synchronization error estimation algorithms are provided.

Finally, we used these joint-estimation algorithms to estimate both offsets and the filtered results are respectively sent to a phase de-rotator for carrier-frequency compensation and a timedomain interpolator for timing error correction, as shown in Fig. 8. These two feedback loops work continuously and cooperatively to maintain system synchronization. The inverses of the LS channel estimation results are used in a simple one-tap equalizer. The constellation of 64-QAM, which is more sensitive to synchronization errors [18], is used as the transmitted frequency-domain signals. The simulated bit-error rate (BER) using different estimation algorithms are plotted in Fig. 9. The SNR degradation of the Sliskovic's algorithm is more than 2 $\mathrm{dB}$ and that of the LLS estimation algorithm is about $0.5 \mathrm{~dB}$. Although previously the LLS algorithm can perform better than the SWLS algorithm in high SNR cases, the close-loop BER results are quite the opposite. This is because, in the close-loop simulation, the residual synchronization errors are much smaller than the previous simulation. Then, the rms errors of the SWLS algorithm approach those of the WLS algorithm, since the ICI effect becomes less significant as the synchronization errors get

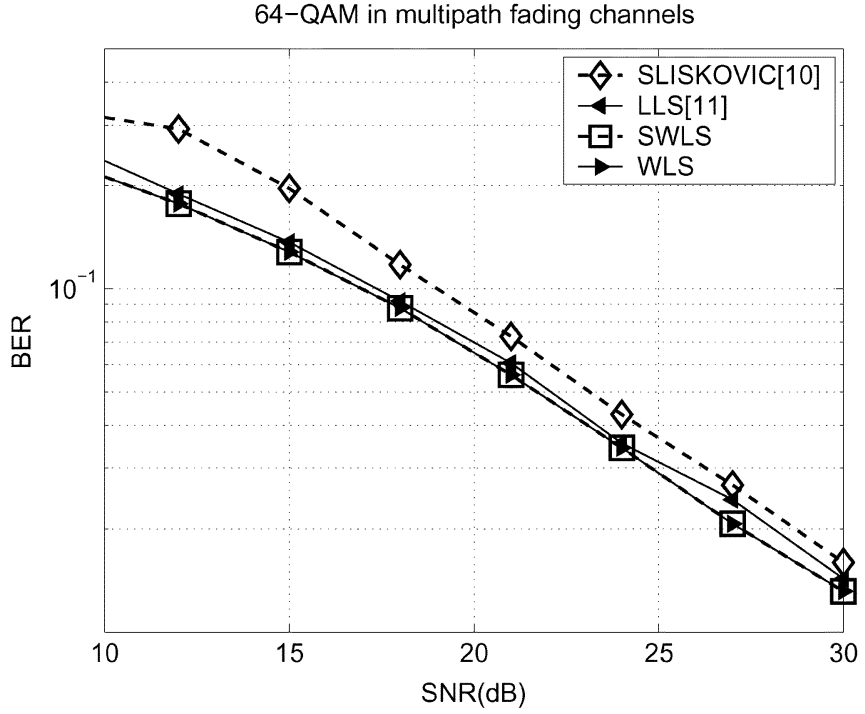

Fig. 9. Error-rate performance of receivers using different joint-estimation algorithms.

smaller. Therefore, the two WLS-based algorithms achieve the same BER performance, which is better than that of the LLS algorithm.

\section{CONCLUSION}

In this paper, we proposed a joint WLS algorithm that can estimate both the carrier-frequency and timing offsets in OFDM signals. We also derived a set of optimal weights and analyzed the proposed algorithm's performance. With the weighted leastsquares technique, the proposed algorithm indeed generates estimates that have minimum rms errors. Simulation results indicate that the Gaussian approximation of the observation variable $\mathbf{y}$ is valid; thus, the proposed WLS algorithm approaches the Cramér-Rao bound. For computational simplicity, a set of simplified weights were introduced and they result in only moderate performance loss in estimation. When used in the tracking mode, the simplified WLS algorithm achieves almost the same performance as the original WLS algorithm and they both outperform the other joint-estimation algorithms. Therefore, the proposed WLS joint-estimation algorithm can greatly enhance the performance of OFDM-based communication receivers by reducing residual synchronization error, thus suppressing intercarrier interference. 


\section{APPENDIX A}

$$
\text { MEAN OF } C_{x_{j}, i} Z_{x_{j}, i} Z_{x_{j}, i-1}^{*}
$$

The means of two terms in (20) are given by

$$
\begin{aligned}
E\{ & \left.C_{x_{j}, i} S_{x_{j}, i} S_{x_{j}, i-1}^{*}\right\} \\
= & E\left\{C_{x_{j}, i} A_{x_{j}, i} H_{x_{j}} s\left(\pi \phi_{x_{j} x_{j}}\right) \cdot e^{j 2 \pi \frac{i N+i N_{g}+N_{g}}{N} \phi_{x_{j} x_{j}}}\right. \\
& \cdot e^{j \pi \phi_{x_{j} x_{j}}\left(1-\frac{1}{N}\right)} \cdot A_{x_{j}, i-1}^{*} H_{x_{j}}^{*} s\left(\pi \phi_{x_{j} x_{j}}\right) \\
& \cdot e^{\left.-j 2 \pi \frac{(i-1) N+(i-1) N_{g}+N_{g}}{N} \phi_{x_{j} x_{j}} \cdot e^{-j \pi \phi_{x_{j} x_{j}}\left(1-\frac{1}{N}\right)}\right\}} \\
= & E_{s}\left|H_{x_{j}}\right|^{2} s^{2}\left(\pi \phi_{x_{j} x_{j}}\right) e^{j 2 \pi \frac{N+N_{g}}{N} \phi_{x_{j} x_{j}}} \\
E\{ & \left.C_{x_{j}, i} S_{x_{j}, i} I_{x_{j}, i-1}^{*}\right\} \\
= & E\left\{C_{x_{j}, i} A_{x_{j}, i} H_{x_{j}} s\left(\pi \phi_{x_{j} x_{j}}\right) \cdot e^{j 2 \pi \frac{i N+i N_{g}+N_{g}}{N} \phi_{x_{j} x_{j}}}\right. \\
& \cdot e^{j \pi \phi_{x_{j} x_{j}}\left(1-\frac{1}{N}\right)} \cdot \sum_{k \neq x_{j}} A_{k, i-1}^{*} H_{k}^{*} s\left(\pi \phi_{k x_{j}}\right) \\
& \left.\cdot e^{-j 2 \pi \frac{(i-1) N+(i-1) N_{g}+N_{g}}{N} \phi_{k k}} \cdot e^{-j \pi \phi_{k x_{j}}\left(1-\frac{1}{N}\right)}\right\} \\
= & K \sum_{k \neq x_{j}} E\left\{C_{x_{j}, i} A_{x_{j}, i} A_{k, i-1}^{*}\right\}=0
\end{aligned}
$$

where $K$ is a complex constant. Similarly, $E\left\{C_{x_{j}, i} I_{x_{j}, i} S_{x_{j}, i-1}^{*}\right\}=0$. The correlation of ICI terms becomes

$$
\begin{aligned}
E\{ & \left.C_{x_{j}, i} I_{x_{j}, i} I_{x_{j}, i-1}^{*}\right\} \\
= & \sum_{k \neq x_{j}} \sum_{q \neq x_{j}} E\left\{C_{x_{j}, i} A_{k, i} A_{q, i-1}^{*}\right\} H_{k} H_{q}^{*} \\
& \cdot s\left(\pi \phi_{k x_{j}}\right) s\left(\pi \phi_{q x_{j}}\right) \cdot e^{j 2 \pi \frac{i N+i N_{g}+N_{g}}{N}\left(\phi_{k k}-\phi_{q q}\right)} \\
& \cdot e^{j \pi\left(1-\frac{1}{N}\right)\left(\phi_{k x_{j}}-\phi_{q x_{j}}\right)} e^{j 2 \pi \frac{N+N_{g}}{N} \phi_{q q}}=0 .
\end{aligned}
$$

Finally, since the data and the AWGN are independent, the expectations of those terms related to the AWGN, $V_{x_{j}, i}$, or $V_{x_{j}, i-1}$ are zero.

$$
\begin{aligned}
E & \left\{C_{x_{j}, i} S_{x_{j}, i} V_{x_{j}, i-1}^{*}\right\} \\
& =E\left\{C_{x_{j}, i} I_{x_{j}, i} V_{x_{j}, i-1}^{*}\right\} \\
& =E\left\{C_{x_{j}, i} V_{x_{j}, i} S_{x_{j}, i-1}^{*}\right\} \\
& =E\left\{C_{x_{j}, i} V_{x_{j}, i} I_{x_{j}, i-1}^{*}\right\} \\
& =E\left\{C_{x_{j}, i} V_{x_{j}, i} V_{x_{j}, i-1}^{*}\right\} \\
& =0 .
\end{aligned}
$$

\section{APPENDIX B \\ VARIANCE OF $C_{x_{j}, i} Z_{x_{j}, i} Z_{x_{j}, i-1}^{*}$}

If $U_{1}, U_{2}, \ldots U_{n}$ are $n$ random variables each having a finite second moment, then the variance of their sum is given by

$$
\operatorname{Var}\left(\sum_{p=1}^{n} U_{p}\right)=\sum_{p=1}^{n} \operatorname{Var}\left(U_{p}\right)+\sum_{p=1}^{n} \sum_{q=1, q \neq p}^{n} \operatorname{Cov}\left(U_{p}, U_{q}\right)
$$

where

$$
\operatorname{Cov}\left(U_{p}, U_{q}\right)=E\left\{\left(U_{p}-E\left\{U_{p}\right\}\right)\left(U_{q}^{*}-E\left\{U_{q}^{*}\right\}\right)\right\} .
$$

From (20), there are nine terms in $C_{x_{j}, i} Z_{x_{j}, i} Z_{x_{j}, i-1}^{*}$; thus, nine variance terms and $9 \times(9-1)=72$ covariance terms. Owing to conjugate symmetry, we only need to compute half of these covariance terms. We will go through these covariance terms by picking one term in (20) and then calculating its covariance with the other terms that follow this term in (20).

First of all, since $C_{x_{j}, i} S_{x_{j}, i} S_{x_{j}, i-1}^{*}$ is deterministic, the covariances of $C_{x_{j}, i} S_{x_{j}, i} S_{x_{j}, i-1}^{*}$ and the other eight terms are all zero. Next, we proceed to the covariances of $C_{x_{j}, i} S_{x_{j}, i} I_{x_{j}, i-1}^{*}$ and the terms that follow it. Previously, we have shown that the expectations of the last eight term in (20) are all zero; thus, the covariance of any pair of these eight terms is equal to their correlation. Because the noise terms are independent of the data, we have

$$
\begin{aligned}
E & \left\{C_{x_{j}, i} S_{x_{j}, i} I_{x_{j}, i-1}^{*} C_{x_{j}, i} S_{x_{j}, i}^{*} V_{x_{j}, i-1}\right\} \\
& =E\left\{C_{x_{j}, i} S_{x_{j}, i} I_{x_{j}, i-1}^{*} C_{x_{j}, i} I_{x_{j}, i}^{*} V_{x_{j}, i-1}\right\} \\
& =E\left\{C_{x_{j}, i} S_{x_{j}, i} I_{x_{j}, i-1}^{*} C_{x_{j}, i} V_{x_{j}, i}^{*} S_{x_{j}, i-1}\right\} \\
& =E\left\{C_{x_{j}, i} S_{x_{j}, i} I_{x_{j}, i-1}^{*} C_{x_{j}, i} V_{x_{j}, i}^{*} I_{x_{j}, i-1}\right\} \\
& =E\left\{C_{x_{j}, i} S_{x_{j}, i} I_{x_{j}, i-1}^{*} C_{x_{j}, i} V_{x_{j}, i}^{*} V_{x_{j}, i-1}\right\} \\
& =0 .
\end{aligned}
$$

Also, since the data are i.i.d., thus

$$
\begin{aligned}
& E \\
& \quad\left\{C_{x_{j}, i} S_{x_{j}, i} I_{x_{j}, i-1}^{*} C_{x_{j}, i} I_{x_{j}, i}^{*} S_{x_{j}, i-1}\right\} \\
& \quad=B_{1} \sum_{k \neq x_{j}} \sum_{q \neq x_{j}} E\left\{C_{x_{j}, i} A_{x_{j}, i} A_{k, i-1}^{*} C_{x_{j}, i} A_{q, i}^{*} A_{x_{j}, i-1}\right\} \\
& \quad=0 \\
& \begin{aligned}
E & \left\{C_{x_{j}, i} S_{x_{j}, i} I_{x_{j}, i-1}^{*} C_{x_{j}, i} I_{x_{j}, i}^{*} I_{x_{j}, i-1}\right\} \\
& =B_{2} \sum_{k \neq x_{j}} \sum_{q \neq x_{j}} \sum_{r \neq x_{j}} E\left\{C_{x_{j}, i} A_{x_{j}, i} A_{k, i-1}^{*} C_{x_{j}, i} A_{q, i}^{*} A_{r, i-1}\right\} \\
& =0
\end{aligned}
\end{aligned}
$$

where $B_{1}$ and $B_{2}$ are complex constants.

We now turn to compute the correlations of $C_{x_{j}, i} S_{x_{j}, i} V_{x_{j}, i-1}^{*}$ and the six terms that follow it. Again, due to the existence of the noise term $V_{x_{j}, i-1}$, any correlation whose other term does not have $V_{x_{j}, i-1}$ is zero. Therefore, we need to compute only

$$
\begin{aligned}
& E\left\{C_{x_{j}, i} S_{x_{j}, i} V_{x_{j}, i-1}^{*} C_{x_{j}, i} I_{x_{j}, i}^{*} V_{x_{j}, i-1}\right\} \\
& =B_{3} \sum_{k \neq x_{j}} E\left\{A_{x_{j}, i} A_{k, i}^{*}\right\} \cdot \sigma_{v}^{2}=0 \\
& E\left\{C_{x_{j}, i} S_{x_{j}, i} V_{x_{j}, i-1}^{*} C_{x_{j}, i} V_{x_{j}, i}^{*} V_{x_{j}, i-1}\right\} \\
& =E\left\{S_{x_{j}, i}\right\} E\left\{V_{x_{j}, i}^{*}\right\} \cdot \sigma_{v}^{2}=0
\end{aligned}
$$

where $B_{3}$ is a complex constant.

When computing the correlations of $C_{x_{j}, i} I_{x_{j}, i} S_{x_{j}, i-1}^{*}$ and the following five terms, we need to evaluate only 
$E\left\{C_{x_{j}, i} I_{x_{j}, i} S_{x_{j}, i-1}^{*} C_{x_{j}, i} I_{x_{j}, i}^{*} I_{x_{j}, i-1}\right\}$, since all other correlations will be zero for having either $V_{x_{j}, i}$ or $V_{x_{j}, i-1}$ and

$$
\begin{aligned}
E & \left\{C_{x_{j}, i} I_{x_{j}, i} S_{x_{j}, i-1}^{*} C_{x_{j}, i} I_{x_{j}, i}^{*} I_{x_{j}, i-1}\right\} \\
& =B_{4} \sum_{k \neq x_{j}} \sum_{q \neq x_{j}} \sum_{r \neq x_{j}} E\left\{C_{x_{j}, i} A_{k, i} A_{x_{j}, i-1}^{*} C_{x_{j}, i} A_{q, i}^{*} A_{r, i-1}\right\} \\
& =0
\end{aligned}
$$

where $B_{4}$ is a complex constant.

As to the correlations of $C_{x_{j}, i} I_{x_{j}, i} I_{x_{j}, i-1}^{*}$ and its following four terms, since all four correlations have at least one noise term, thus all four correlations are zero. Similarly, the remaining six correlations that involve $C_{x_{j}, i} I_{x_{j}, i} V_{x_{j}, i-1}^{*}, C_{x_{j}, i} V_{x_{j}, i} S_{x_{j}, i-1}^{*}, C_{x_{j}, i} V_{x_{j}, i} I_{x_{j}, i-1}^{*}$, and $C_{x_{j}, i} V_{x_{j}, i} V_{x_{j}, i-1}^{*}$ are all zero. In conclusion, all 72 covariances are zero and the variance of $C_{x_{j}, i} Z_{x_{j}, i} Z_{x_{j}, i-1}^{*}$ is the sum of the variances of individual terms in (20).

Next, we turn to the variance of each term in (20). The variance of $C_{x_{j}, i} S_{x_{j}, i} S_{x_{j}, i-1}^{*}$ is zero since it is deterministic. For the variance of either product of the signal term and the ICI term, we have

$$
\begin{aligned}
\operatorname{Var} & \left\{C_{x_{j}, i} S_{x_{j}, i} I_{x_{j}, i-1}^{*}\right\} \\
& =\operatorname{Var}\left\{C_{x_{j}, i} I_{x_{j}, i} S_{x_{j}, i-1}^{*}\right\} \\
& =E_{s}\left|H_{x_{j}}\right|^{2} s^{2}\left(\pi \phi_{x_{j} x_{j}}\right) \cdot \sum_{k \neq x_{j}} E_{s}\left|H_{k}\right|^{2} s^{2}\left(\pi \phi_{k x_{j}}\right)
\end{aligned}
$$

and the variances of products of the signal term and the noise term are

$$
\begin{aligned}
& \operatorname{Var}\left\{C_{x_{j}, i} S_{x_{j}, i} V_{x_{j}, i-1}^{*}\right\} \\
& =\operatorname{Var}\left\{C_{x_{j}, i} V_{x_{j}, i} S_{x_{j}, i-1}^{*}\right\} \\
& =E_{s}\left|H_{x_{j}}\right|^{2} s^{2}\left(\pi \phi_{x_{j} x_{j}}\right) \cdot \sigma_{v}^{2}
\end{aligned}
$$

The variances of the remaining four terms can be similarly derived as

$$
\begin{aligned}
& \operatorname{Var}\left\{C_{x_{j}, i} I_{x_{j}, i} V_{x_{j}, i-1}^{*}\right\}=\operatorname{Var}\left\{C_{x_{j}, i} V_{x_{j}, i} I_{x_{j}, i-1}^{*}\right\} \\
& =\sum_{k \neq x_{j}} E_{s}\left|H_{k}\right|^{2} s^{2}\left(\pi \phi_{k x_{j}}\right) \cdot \sigma_{v}^{2}, \\
& \operatorname{Var}\left\{C_{x_{j}, i} I_{x_{j}, i} I_{x_{j}, i-1}^{*}\right\} \\
& =\left(\sum_{k \neq x_{j}} E_{s}\left|H_{k}\right|^{2} s^{2}\left(\pi \phi_{k x_{j}}\right)\right)^{2}
\end{aligned}
$$

and

$$
\operatorname{Var}\left\{C_{x_{j}, i} V_{x_{j}, i} V_{x_{j}, i-1}^{*}\right\}=\sigma_{v}^{4} .
$$

In the case of moderately high SINR, when the ICI term is attenuated by the factor $s\left(\pi \phi_{k x_{j}}\right)$ for $k \neq j$, the variance of $C_{x_{j}, i} Z_{x_{j}, i} Z_{x_{j}, i-1}^{*}$ is dominated by the variances of those terms with either the signal $S_{x_{j}, i}$ or $S_{x_{j}, i-1}$.

\section{APPENDIX C}

COVARIANCE OF $C_{x_{j}, i} Z_{x_{j}, i} Z_{x_{j}, i-1}^{*}$ and $C_{x_{l}, i} Z_{x_{l}, i} Z_{x_{l}, i-1}^{*}$

Similar to the derivation in Appendix B, the covariance of $C_{x_{j}, i} Z_{x_{j}, i} Z_{x_{j}, i-1}^{*}$ and $C_{x_{l}, i} Z_{x_{l}, i} Z_{x_{l}, i-1}^{*}$ can be expressed as the sum of 81 covariances. Since all four noise variables, $V_{x_{j}, i}, V_{x_{j}, i-1}, V_{x_{l}, i}$, and $V_{x_{l}, i-1}$, are mutually independent and are independent of the data, all terms having at least one noise variable will vanish. Therefore, we have

$$
\begin{aligned}
\operatorname{Cov} & \left(C_{x_{j}, i} Z_{x_{j}, i} Z_{x_{j}, i-1}^{*}, C_{x_{l}, i} Z_{x_{l}, i} Z_{x_{l}, i-1}^{*}\right) \\
= & E\left\{C_{x_{j}, i}\left(Z_{x_{j}, i} Z_{x_{j}, i-1}^{*}-S_{x_{j}, i} S_{x_{j}, i-1}^{*}\right)\right. \\
& \left.\cdot C_{x_{l}, i}\left(Z_{x_{l}, i} Z_{x_{l}, i-1}^{*}-S_{x_{l}, i} S_{x_{l}, i-1}^{*}\right)^{*}\right\} \\
= & E\left\{C_{x_{j}, i}\left(S_{x_{j}, i} I_{x_{j}, i-1}^{*}\right) C_{x_{l}, i}\left(S_{x_{l}, i} I_{x_{l}, i-1}^{*}\right)^{*}\right\} \\
& +E\left\{C_{x_{j}, i}\left(S_{x_{j}, i} I_{x_{j}, i-1}^{*}\right) C_{x_{l}, i}\left(I_{x_{l}, i} S_{x_{l}, i-1}^{*}\right)^{*}\right\} \\
& +E\left\{C_{x_{j}, i}\left(S_{x_{j}, i} I_{x_{j}, i-1}^{*}\right) C_{x_{l}, i}\left(I_{x_{l}, i} I_{x_{l}, i-1}^{*}\right)^{*}\right\} \\
& +E\left\{C_{x_{j}, i}\left(I_{x_{j}, i} S_{x_{j}, i-1}^{*}\right) C_{x_{l}, i}\left(S_{x_{l}, i} I_{x_{l}, i-1}^{*}\right)^{*}\right\} \\
& +E\left\{C_{x_{j}, i}\left(I_{x_{j}, i} S_{x_{j}, i-1}^{*}\right) C_{x_{l}, i}\left(I_{x_{l}, i} S_{x_{l}, i-1}^{*}\right)^{*}\right\} \\
& +E\left\{C_{x_{j}, i}\left(I_{x_{j}, i} S_{x_{j}, i-1}^{*}\right) C_{x_{l}, i}\left(I_{x_{l}, i} I_{x_{l}, i-1}^{*}\right)^{*}\right\} \\
& +E\left\{C_{x_{j}, i}\left(I_{x_{j}, i} I_{x_{j}, i-1}^{*}\right) C_{x_{l}, i}\left(S_{x_{l}, i} I_{x_{l}, i-1}^{*}\right)^{*}\right\} \\
& +E\left\{C_{x_{j}, i}\left(I_{x_{j}, i} I_{x_{j}, i-1}^{*}\right) C_{x_{l}, i}\left(I_{x_{l}, i} S_{x_{l}, i-1}^{*}\right)^{*}\right\} \\
& +E\left\{C_{x_{j}, i}\left(I_{x_{j}, i} I_{x_{j}, i-1}^{*}\right) C_{x_{l}, i}\left(I_{x_{l}, i} I_{x_{l}, i-1}^{*}\right)^{*}\right\} .
\end{aligned}
$$

For clarity, the effects of phase rotation, magnitude attenuation, and channel complex gain in the nine terms will all be absorbed into constants and denoted as $K_{p}, p=1, \ldots, 9$. Note that the first term and the fifth term are similar and

$$
\begin{aligned}
E & \left\{C_{x_{j}, i} S_{x_{j}, i} I_{x_{j}, i-1}^{*} C_{x_{l}, i} S_{x_{l}, i}^{*} I_{x_{l}, i-1}\right\} \\
& =K_{1} \sum_{k \neq x_{j}} \sum_{q \neq x_{l}} E\left\{C_{x_{j}, i} A_{x_{j}, i} A_{k, i-1}^{*} C_{x_{l}, i} A_{x_{l}, i}^{*} A_{q, i-1}\right\} \\
& =0 \\
E & \left\{C_{x_{j}, i} I_{x_{j}, i} S_{x_{j}, i-1}^{*} C_{x_{l}, i} I_{x_{l}, i}^{*} S_{x_{l}, i-1}\right\} \\
& =K_{5} \sum_{k \neq x_{j}} \sum_{q \neq x_{l}} E\left\{C_{x_{j}, i} A_{k, i} A_{x_{j}, i-1}^{*} C_{x_{l}, i} A_{q, i}^{*} A_{x_{l}, i-1}\right\} \\
& =0 .
\end{aligned}
$$

Likewise, the second and fourth terms can be evaluated and

$$
\begin{aligned}
E & \left\{C_{x_{j}, i} S_{x_{j}, i} I_{x_{j}, i-1}^{*} C_{x_{l}, i} I_{x_{l}, i}^{*} S_{x_{l}, i-1}\right\} \\
& =K_{2} \sum_{k \neq x_{j}} \sum_{q \neq x_{l}} E\left\{C_{x_{j}, i} A_{x_{j}, i} A_{k, i-1}^{*} C_{x_{l}, i} A_{q, i}^{*} A_{x_{l}, i-1}\right\} \\
& =0 \\
E & \left\{C_{x_{j}, i} I_{x_{j}, i} S_{x_{j}, i-1}^{*} C_{x_{l}, i} S_{x_{l}, i}^{*} I_{x_{l}, i-1}\right\} \\
& =K_{4} \sum_{k \neq x_{j}} \sum_{q \neq x_{l}} E\left\{C_{x_{j}, i} A_{k, i} A_{x_{j}, i-1}^{*} C_{x_{l}, i} A_{x_{l}, i}^{*} A_{q, i-1}\right\} \\
& =0 .
\end{aligned}
$$


The third and sixth terms are similar and are also equal to zero.

$$
\begin{aligned}
& E\left\{C_{x_{j}, i} S_{x_{j}, i} I_{x_{j}, i-1}^{*} C_{x_{l}, i} I_{x_{l}, i}^{*} I_{x_{l}, i-1}\right\} \\
& =K_{3} \sum_{k \neq x_{j}} \sum_{q \neq x_{l}} \sum_{r \neq x_{l}} E\left\{C_{x_{j}, i} A_{x_{j}, i} A_{k, i-1}^{*} C_{x_{l}, i} A_{q, i}^{*} A_{r, i-1}\right\} \\
& \quad=0 \\
& E
\end{aligned}
$$

For the seventh and eighth terms, we have

$$
\begin{aligned}
& E\left\{C_{x_{j}, i} I_{x_{j}, i} I_{x_{j}, i-1}^{*} C_{x_{l}, i} S_{x_{l}, i}^{*} I_{x_{l}, i-1}\right\} \\
& \quad=K_{7} \sum_{k \neq x_{j}} \sum_{q \neq x_{j}} \sum_{r \neq x_{l}} E\left\{C_{x_{j}, i} A_{k, i} A_{q, i-1}^{*} C_{x_{l}, i} A_{x_{l}, i}^{*} A_{r, i-1}\right\} \\
& \quad=0 \\
& E
\end{aligned}
$$

Finally, the ninth term is nonzero and

$$
\begin{aligned}
E & \left\{C_{x_{j}, i} I_{x_{j}, i} I_{x_{j}, i-1}^{*} C_{x_{l}, i} I_{x_{l}, i}^{*} I_{x_{l}, i-1}\right\} \\
= & K_{9} \sum_{k \neq x_{j}} \sum_{p \neq x_{j}} \sum_{q \neq x_{l}} \sum_{r \neq x_{l}} E\left\{C_{x_{j}, i} A_{k, i} A_{p, i-1}^{*} C_{x_{l}, i} A_{q, i}^{*} A_{r, i-1}\right\} \\
= & K_{9} \sum_{k \neq x_{j}} \sum_{p \neq x_{j}} \sum_{q \neq x_{l}} \sum_{r \neq x_{l}} E_{s}^{2} \delta_{x_{l} k} \delta_{x_{l} p} \delta_{x_{j} q} \delta_{x_{j} r} \\
= & E_{s}^{2} s^{2}\left(\pi \phi_{x_{l} x_{j}}\right) s^{2}\left(\pi \phi_{x_{j} x_{l}}\right)\left|H_{x_{j}}\right|^{2}\left|H_{x_{l}}\right|^{2} \\
& \cdot e^{j 2 \pi \frac{N+N_{g}}{N} \phi_{x_{l} x_{l}}} e^{j 2 \pi \frac{N+N_{g}}{N} \phi_{x_{j} x_{j}}} .
\end{aligned}
$$

In summary

$$
\begin{aligned}
\operatorname{Cov} & \left(C_{x_{j}, i} Z_{x_{j}, i} Z_{x_{j}, i-1}^{*}, C_{x_{l}, i} Z_{x_{l}, i} Z_{x_{l}, i-1}^{*}\right) \\
= & E_{s}^{2} s^{2}\left(\pi \phi_{x_{l} x_{j}}\right) s^{2}\left(\pi \phi_{x_{j} x_{l}}\right)\left|H_{x_{j}}\right|^{2}\left|H_{x_{l}}\right|^{2} \\
& \cdot e^{j 2 \pi \frac{N+N_{g}}{N} \phi_{x_{l} x_{l}}} e^{j 2 \pi \frac{N+N_{g}}{N} \phi_{x_{j} x_{j}}} .
\end{aligned}
$$

This value is quite small because the attenuation of $s^{2}\left(\pi \phi_{x_{l} x_{j}}\right)$ and $s^{2}\left(\pi \phi_{x_{j} x_{l}}\right)$ is significant due to the pilot spacing between $x_{l}$ and $x_{j}$. Compared to $\operatorname{Var}\left(C_{x_{j}, i} Z_{x_{j}, i} Z_{x_{j}, i-1}^{*}\right)$ and $\operatorname{Var}\left(C_{x_{l}, i} Z_{x_{l}, i} Z_{x_{l}, i-1}^{*}\right)$, this covariance is negligible.

\section{REFERENCES}

[1] F. Classen and H. Meyr, "Frequency synchronization algorithms for OFDM systems suitable for communication over frequency selective fading channels," in Proc. IEEE VTC'94, Stockholm, Sweden, Jun. 1994, pp. 1655-1659.
[2] S. Kapoor, D. J. Marchok, and Y.-F. Huang, "Pilot assisted synchronization for wireless OFDM systems over fast time varying fading channels," in Proc. IEEE VTC'98, Ottawa, ON, Canada, May 1998, pp. 2077-2080.

[3] P. H. Moose, "A technique for orthogonal frequency division multiplexing frequency offset correction," IEEE Trans. Commun., vol. 42, no. 10, pp. 2908-2914, Oct. 1994.

[4] D. K. Kim, S. H. Do, H. B. Cho, H. J. Choi, and K. B. Kim, "A new joint algorithm of symbol timing recovery and sampling clock adjustment for OFDM systems," IEEE Trans. Consumer Electron., vol. 44, no. 3, pp. 1142-1149, Aug. 1998.

[5] Y.-J. Ryu and D.-S. Han, "Timing phase estimator overcoming Rayleigh fading for OFDM systems," IEEE Trans. Consumer Electron., vol. 47, no. 3, pp. 370-377, Aug. 2001.

[6] J. H. Yooh and V. K. Wei, "On synchronizing and detecting multi-carrier CDMA signals," in Proc. IEEE Int. Conf. Universal Personal Communications, Tokyo, Japan, Nov. 1995, pp. 512-516.

[7] A. J. Coulson, "Maximum likelihood synchronization for OFDM using a pilot: Algorithms," IEEE J. Select. Areas Commun., vol. 19, no. 12, pp. 2486-2494, Dec. 2001.

[8] I. H. Hwang, H. S. Lee, and K. W. Kang, "Frequency and timing period offset estimation technique for OFDM systems," Electron. Lett., vol. 34, no. 6, pp. 520-521, 2001.

[9] S. Simoens, V. Buzenac, and M. D. Courville, "A new method for joint cancellation of clock and carrier frequency offsets in OFDM receivers over frequency selective channels," in Proc. IEEE VTC'00, Tokyo, Japan, May 2000, pp. 390-394.

[10] M. Sliskovic, "Carrier and Sampling frequency offset estimation and correction in multicarrier systems," in Proc. IEEE GLOBECOM'01, San Antonio, TX, Nov. 2001, pp. 285-289.

[11] S. Y. Liu and J. W. Chong, "A study of joint tracking algorithms of carrier frequency offset and sampling clock offset for OFDM-based WLANs," in IEEE 2002 Int. Conf. Communications, Circuits and Systems and West Sino Expositions, Chengdu City, China, Jun. 2002, pp. 109-113.

[12] L. L. Scharf, Statistical Signal Processing, Detection, Estimation, and Time Series Analysis. Boston, MA: Addison-Wesley, 1991.

[13] A. J. Coulson, "Maximum likelihood synchronization for OFDM using a pilot: Analysis," IEEE J. Select. Areas Commun., vol. 19, no. 12, pp. 2495-2503, Dec. 2001.

[14] X. Wang, T. T. Tjhung, Y. Wu, and B. Caron, "SER performance evaluation and optimization of OFDM system with residual frequency and timing offsets from imperfect synchronization," IEEE Trans. Broadcast., vol. 49, no. 2, pp. 170-177, Jun. 2003.

[15] T. K. Moon and W. C. Stirling, Mathematical Methods and Algorithms for Signal Processing. Englewood Cliffs, NJ: Prentice-Hall, 2000.

[16] 3GPP Technique Specification Group (TSG) RAN WG4; Deployment Aspects. TR 25.943 v2.1.0 (2001-06).

[17] P. Y. Tsai, H. Y. Kang, and T. D. Chiueh, "Joint weighted least squares estimation of frequency and timing offset for OFDM systems over fading channels," in Proc. IEEE VTC'03, Cheju, Korea, Apr. 2003, pp. 2543-2547.

[18] T. Pollet, M. Van Bladel, and M. Moeneclaey, "BER sensitivity of OFDM systems to carrier frequency offset and Wiener phase noise," IEEE Trans. Commun., vol. 43, no. 2/3/4, pp. 191-193, Feb./Mar./Apr. 1995.

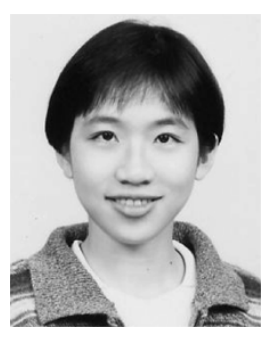

Pei-Yun Tsai (S'02) was born in Taipei, Taiwan, on May 11, 1972. She received the B.S. and M.S. degree in electrical engineering from the National Taiwan University, Taipei, Taiwan, in 1994 and 1996, respectively, and is currently working toward the Ph.D. degree in electrical engineering at National Taiwan University.

From 1996 to 2000, she was with ASUStek, Taipei, Taiwan, and participated in the team of optical storage systems. Her research interests include base-band signal-processing algorithms and integrated circuit design for digital communication systems. 


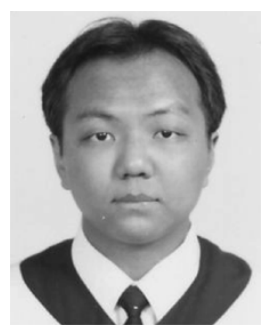

Hsin-Yu Kang was born in Taipei, Taiwan, in 1979. $\mathrm{He}$ received the B.S. degree in electrical engineering and the M.S. degree in electronics engineering from National Taiwan University, Taipei, Taiwan, in 2001 and 2003, respectively.

His research interests include base-band signal processing of communication systems and related very large scale integration (VLSI) design.

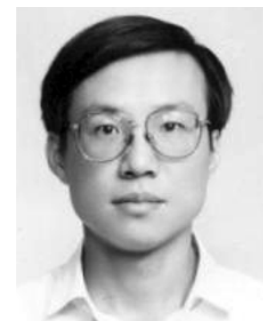

Tzi-Dar Chiueh (S'87-M'90-SM'03) was born in Taipei, Taiwan, on July 3, 1960. He received the B.S.E.E. degree from the National Taiwan University, Taipei, Taiwan, in 1983 and the M.S. and Ph.D. degrees in electrical engineering from the California Institute of Technology, Pasadena, in 1986 and 1989, respectively.

Since 1989, he has been with the Department of Electrical Engineering, National Taiwan University, where he currently is a Professor. He became Director of the Graduate Institute of Electronics Engineering, National Taiwan University, in August 2004. His research interests include integrated circuit design for digital communication systems and other signal-processing systems. 